\title{
Balloon Plasty of Pseudoaneurysm of AV Fistula in CKD
}

\author{
Anusha Gupta ${ }^{1}$, Sourya Acharya ${ }^{2}$, Deep Hathi ${ }^{3}$ \\ ${ }^{1}$ Department of General Medicine, Datta Meghe Institute of Medical Sciences, Wardha, Maharashtra, India. \\ 2Department of General Medicine, Datta Meghe Institute of Medical Sciences, Wardha, Maharashtra, India. \\ ${ }^{3}$ Department of General Medicine, Datta Meghe Institute of Medical Sciences, Wardha, Maharashtra, India.
}

\section{INTRODUCTION}

Arteriovenous fistula is a procedure done for long-term haemodialysis in patients with end stage renal failure. Aneurysms are a common and often difficult complication seen with arterio venous vascular access for haemodialysis. In this case report we describe middle aged male patient who was a known case of chronic kidney disease on maintenance haemodialysis through left arm brachio-basilic AV fistula and had developed a pseudo aneurysm of the AVF. We performed an Endovascular intervention for salvaging haemodialysis AV fistula in which balloon plasty was done and flow was obtained.

To ensure adequate haemodialysis treatments, a well-functioning vascular access (VA) is a prerequisite in dialysis patients. Arteriovenous fistula (AVF) has become a long-term haemodialysis treatment in patients with renal failure in the end stage and this has not changed until now.(1) For long-term haemodialysis with low morbidity levels, arteriovenous fistula should provide a convenient repeated vascular access line. The development of aneurysm may occur due to frequent punctures that in some patients may weaken the vein wall. Proximal stenosis often speeds up the process by increasing the pressure in the AVF.(2) Such patients may have a large, painful aneurysm sac, which can lead to dermal tissue necrosis. It may become compromised as a result of vascular or traumatic events. The aneurysm sac can split, which may result in fatal haemorrhage.

\section{PRESENTATION OF CASE}

A 53 years old male patient, a known case of chronic kidney disease on maintenance haemodialysis for the past 3 months, who had a left arm A-V fistula, was admitted to the hospital with the complaints of sudden onset fever with chills along with pain and swelling over the left arm (at the site of A-V fistula) for the past 3 days. He also had complaints of nausea, vomiting and breathlessness. The patient was a known case of systemic hypertension and was not compliant with the treatment. He was also a known case of Diabetes Mellitus type II and was on treatment for the same. On examination, there was tenderness and raised temperature over the AV fistula site. The patient's haemoglobin was decreased, and his creatinine was elevated. His WBC count was raised. He was subsequently started on higher antibiotics. In view of low haemoglobin level, the patient was given blood transfusion. Alternatively, a triple lumen dialysis cannula was inserted in the right internal jugular vein of the patient and haemodialysis was performed through the same.

\author{
Corresponding Author: \\ Anusha Gupta, \\ Radhika Bai Girls Hotel, \\ Sawangi, Meghe, Wardha, \\ Maharashtra, India. \\ E-mail: dranushagupta92@gmail.com
}

DOI: $10.14260 /$ jemds/2020/302

Financial or Other Competing Interests: None.

How to Cite This Article:

Gupta A, Acharya S, Hathi D. Balloon plasty of pseudoaneurysm of AV fistula in CKD. J. Evolution Med. Dent. Sci. 2020;9(16):13901393, DOI: $10.14260 /$ jemds/2020/302

Submission 02-02-2020,

Peer Review 26-03-2020

Acceptance 03-04-2020,

Published 20-04-2020.

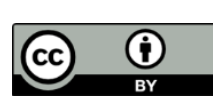


Following three sessions of haemodialysis that was done along with the blood transfusion the patient's condition was stabilized and plan for AVF salvation was undertaken. Colour Doppler of the left upper limb was done that was s/o pseudoaneurysm and haematoma at the venous end of the brachio-basilic AV fistula. Also left upper limb angiography was done that indicated stenosis in basilic vein with multiple flow related pseudoaneurysms. Subsequently endovascular intervention for salvaging haemodialysis AV fistula was performed in which balloon plasty was done and flow was obtained.

\section{DISCUSSION}

Patients with renal failure in the final stage, arteriovenous fistula has become a long-term therapy for haemodialysis. There is a link in the arm between an artery and a vein that strengthens the vein, allowing the positioning of dialysis needles. Also the fistula has a large diameter that allows the blood to flow back and forth into the body quickly. Aneurysms are a common complication seen in haemodialysis with arteriovenous access, which is often difficult.(2) Since the aim of VA formation is to create abnormally dilated vasculature, in this setting it is difficult to define aneurysmal growth. It may be beneficial to classify aneurysms into true and false wallbased aneurysms. The level of aneurysms production is around 5-6 percent.(2) Pseudoaneurysms can be caused by anastomosis and reflect blood leakage outside the lumen perioperative as a result of surgical technique or later occur as an infection complication. Patients may have an uplifting, painful aneurysmal bag that can lead to necrosis of the dermal tissue. Pseudoaneurysm formation is known to be a late complication of AVF that usually occurs after the fistula has matured. For patients with AV fistula for haemodialysis, routine puncture therapy with anticoagulation and irregular haemodynamics makes pseudoaneurysms and true aneurysms a relatively common complication. Most generally, the natural history of VA aneurysms is a benign phase in which patients remain stable and asymptomatic without sacrificing access or haemodialysis. The options for treatment are surgical or radiological, and the desired outcome should be preservation of definitive access where possible.(2) Some procedures such as surgical ligation, resection, stent, and perivascular metal meshes can address the problem of pseudoaneurysm.(3-7)

\section{CONCLUSIONS}

In patients with VA for haemodialysis, regular puncture treatment with anticoagulation and abnormal haemodynamics makes pseudoaneurysms and true aneurysms a relatively common complication. Daily VA monitoring can allow aneurysms to be identified earlier and require prompt care to prevent life-threatening complications; however, there is no research that describes the natural history and development of aneurysms, and it is necessary to identify the best timing of action as well as the optimal modality of treatment.
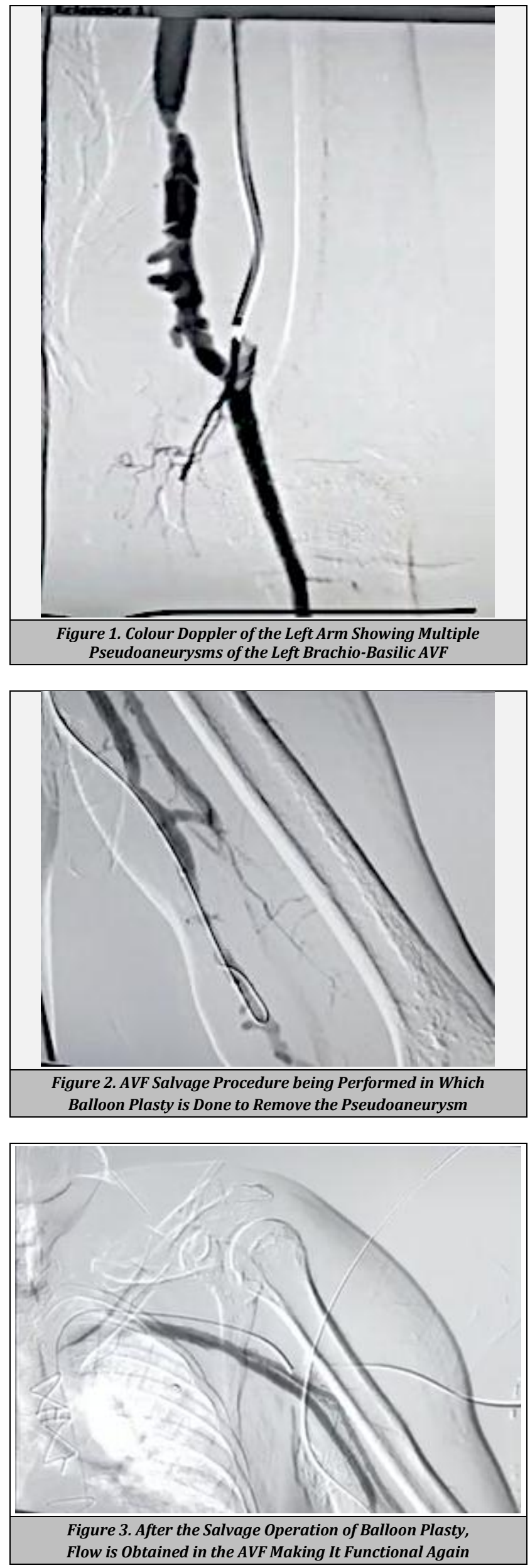


\section{REFERENCES}

[1] Mudoni A, Cornacchiari M, Gallieni M, et al. Aneurysms and pseudoaneurysms in dialysis access. Clin Kidney J 2015;8(4):363-7.

[2] Bachleda P, Utikal P, Zadrazil J, et al. Aneurysm as a complication of arteriovenous anastomoses for haemodialysis. Rozhl Chir 1998;77(12):541-4.

[3] Allaria PM, Costantini E, Lucatello A, et al. Aneurysm of arteriovenous fistula in uremic patients: is endograft a viable therapeutic approach? J Vasc Access 2002;3(2):858.
[4] Karabay 0, Yetkin U, Silistreli E, et al. Surgical management of giant aneurysms complicating arteriovenous fistulae. J Int Med Res 2004;32:214-7.

[5] Grauhan 0, Zurbrugg HR, Hetzer R. Management of aneurysmal arteriovenous fistula by a perivascular metal mesh. Eur J Vasc Endovasc Surg 2001;21(3):274-5.

[6] Lo HY, Tan SG. Arteriovenous fistula aneurysm - plicate, not ligate. Ann Acad Med Singapore 2007;36(10):851-3.

[7] Valentine CS, Aworanti O. Surgical management of aneurysms of arteriovenous fistulae in haemodialysis patients: a case series. Open Access Surgery 2010;3:9-12. 